Laboratorio de Endocrinología y Metabolismo, Facultad de Medicina Occidente, Universidad de Chile.

angeniero en Biotecnología Molecular.

${ }^{\text {b} A l u m n a s ~ d e ~ l a ~ c a r r e r a ~ d e ~}$ Medicina, Universidad de Chile.

Financiado por los Proyectos FONDECYT \# 1030487 ,

\#1071007 y \#1110864

Recibido el 9 de enero de 2014 aceptado el 23 de julio de 2014

Correspondencia a: Dra. T. Sir-Petermann Carlos Schachtebeck (ex Las Palmeras) 299, Interior Quinta Normal

Casilla 33052, Correo 33 Santiago, Chile Teléfono: 56-2-26814676 Fax: 56-2-26816693 tsir@med.uchile.cl

\section{Clasificación de los fenotipos de síndrome de ovario poliquístico de acuerdo a los criterios de Rotterdam: ¿una condición estática o variable?}

\author{
BÁRBARA ECHIBURÚa, AMANDA LADRÓN DE GUEVARA, \\ CECILIA PEREIRA, CONSTANZA PÉREZ ${ }^{\mathrm{b}}$, PÍA MICHAEL ${ }^{\mathrm{b}}$, \\ NICOLÁS CRISOSTO, TERESA SIR-PETERMANN
}

\section{Effects of pregnancy and changes in body weight on polycystic ovary syndrome phenotypes according to the Rotterdam criteria}

Background: Polycystic Ovary Syndrome (PCOS) is tightly associated with insulin resistance and obesity and characterized by hyperandrogenism, chronic oligo-anovulation and polycystic ovarian morphology when fully expressed. The 2003 Rotterdam consensus proposed that two or three of these features were necessary to make the diagnosis, which generated four phenotypes. Several studies have suggested that these phenotypes could differ in their metabolic and endocrine characteristics and that they could vary in the same patient when analyzed throughout life. Aim: To determine if the initial classification of PCOS phenotypes is modified by different physiological conditions. Material and Methods: We performed a non-concurrent prospective analysis of 88 women with PCOS according to the Rotterdam criteria. The effect of physiological conditions such as changes in body weight, pregnancy and ageing more than five years on PCOS phenotype expression was analyzed. Results: Twenty four percent of women became pregnant, $37 \%$ decreased and $24 \%$ increased their body weight during follow up. These conditions modified significantly the proportion of the different phenotypes $\left(\chi^{2}=32.2, p<0.001\right)$. For instance, weight reduction was associated with a change to a better phenotype $(p=0.047)$ and even a normalization of the $P C O S$ condition in $27 \%$ of the patients. On the other hand, an increase in body weight modifying body mass index in one unit, conferred an $8 \%$ probability of changing to a worst phenotype. Conclusions: Pregnancy and changes in body weight significantly modify PCOS phenotypes.

(Rev Med Chile 2014; 142: 966-974)

Key words: Age factors; Obesity; Phenotype; Polycystic ovary syndrome; Pregnancy.
41 1 síndrome de ovario poliquístico (SOP) es una enfermedad endocrino-metabólica $\checkmark$ común en la mujer en edad reproductiva ${ }^{1}$ y afectaría aproximadamente 6-8\% de ellas, dependiendo del criterio de diagnóstico utilizado ${ }^{2}$. EL SOP se caracteriza por hiperandrogenismo, oligo-ovulación crónica y ovarios de aspecto po- liquísticos, además, frecuentemente se asocia con resistencia insulínica (RI) y obesidad. Es probable que este síndrome tenga un origen genético ${ }^{3,4}$, el cual sería influenciado por factores ambientales ${ }^{5,6}$.

Si bien el SOP fue descrito en 1935 por Stein y Leventhal ${ }^{7}$, sigue siendo controvertido debido a su heterogeneidad, lo que ha llevado a plantear 
distintas definiciones. En 1990, en una conferencia de consenso de la National Institutes of Health (NIH) de los Estados Unidos de Norteamérica, se lo definió como la "presencia de hiperandrogenismo asociado a anovulación crónica sin otra causa específica de enfermedad adrenal o hipofisiaria que curse con irregularidades menstruales o exceso de andrógenos" $"$, sin considerar el aspecto morfológico de los ovario. Posteriormente, la Sociedad Europea de Reproducción y Embriología (ESHRE) y la Sociedad Americana de Medicina Reproductiva (ASRM) en una conferencia de consenso realizada en Rotterdam el año 2003, propuso una nueva definición del síndrome que incorporó la presencia de morfología de ovarios poliquísticos (MOP) en la ultrasonografía9. Se planteó, que luego de excluir otras formas de hiperandrogenismo, el SOP podía ser diagnosticado en pacientes que presentaran a lo menos dos de las tres características siguientes: hiperandrogenismo clínico o bioquímico, oligo-anovulación, y MOP, dando origen a cuatro fenotipos. Fenotipo A: oligo o anovulación, hiperandrogenismo y MOP; fenotipo B: oligo ovulación e hiperandrogenismo; fenotipo C: hiperandrogenismo y MOP y fenotipo D: oligo ovulación y MOP. Este fenotipo es controversial ya que no presenta hiperandrogenismo ${ }^{10}$.

Recientemente se ha sugerido que los fenotipos de Rotterdam difieren en sus características metabólicas y endocrinas. Estudios previos han establecido que las mujeres con los fenotipos clásicos de SOP (A y B) estarían más afectadas metabólicamente que los fenotipos $\mathrm{C}$ y $\mathrm{D}^{11-12}$. Sin embargo, otros estudios no han confirmado estos resultados ${ }^{13,14}$.

A lo largo de la etapa reproductiva de estas pacientes, factores ambientales o situaciones fisiológicas podrían afectar la susceptibilidad de expresar un determinado fenotipo. Entre ellas se han mencionado el cambio del peso corporal y el paso del tiempo.

Probablemente la obesidad tendría un impacto negativo en la manifestación del SOP, por estar ligada con hiperandrogenismo y compromiso metabólico ${ }^{15,16}$. Por el contrario, la disminución del peso corporal tendría un efecto favorable, pues reestablecería la ciclicidad menstrual y mejoraría el estado metabólico ${ }^{17}$.

Estudios observacionales han sugerido que algunos de los síntomas y características del SOP podrían mejorar con la edad ${ }^{14,18}$.
Finalmente, un hecho que no ha sido considerado es el impacto que podría tener el período embarazo-lactancia en la expresión de este síndrome, fundamentalmente al término de la lactancia, cuando se reanuda la función ovárica.

El objetivo del presente estudio fue evaluar si la clasificación inicial de los fenotipos de SOP de acuerdo al criterio de Rotterdam se modifica con condiciones fisiológicas, como la variación del peso corporal, la edad y el período embarazo-lactancia.

\section{Pacientes y Método}

Se incluyeron 88 mujeres entre 17 y 36 años, quienes consultaron en nuestro policlínico docente del laboratorio de Endocrinología y Metabolismo de la Facultad de Medicina Occidente de la Universidad de Chile desde el año 1998 en adelante y que fueron catalogadas como portadoras de SOP. Las mujeres que ingresaron entre 1998-2004 fueron diagnosticadas de acuerdo a los criterios de NIH y posteriormente reclasificadas según los fenotipos de Rotterdam. Aquellas que ingresaron después del año 2004 fueron clasificadas desde el inicio de acuerdo a estos fenotipos. Todas las mujeres fueron evaluadas antes y después de las condiciones anteriormente descritas. Dado que ellas han participado en distintos protocolos de nuestro laboratorio, las mediciones antropométricas y bioquímicas fueron hechas en el mismo centro universitario y con los mismos ensayos. Los estudios bioquímicos y ecográficos fueron realizados durante la fase folicular temprana (día 3-8) de un ciclo espontáneo y en pacientes con amenorrea, después de la determinación de progesterona sérica (en rango folicular).

Todas las pacientes contaban con mediciones antropométricas completas: peso, talla, diámetro de cintura y cadera, índice cintura cadera (ICC), índice de masa corporal (IMC), presión arterial y score de Ferriman-Gallwey ${ }^{19}$ modificado por Hatch R et al.

Además, todas ellas tenían una prueba de tolerancia a la glucosa oral (PTGO) con determinación de glucosa e insulina y perfil lipídico en la muestra basal. Las mediciones derivadas de la PTGO fueron: HOMA-IR (homeostasis model assessment for insulin resistance $)^{20}$ e índice de sensibilidad a la insulina (ISI composite) ${ }^{21}$.

Como criterio de inclusión para ingresar al 
Efecto de condiciones fisiológicas sobre los fenotipos de SOP - B. Echiburú et al

estudio se consideró 2 ó 3 de las siguientes características: oligo/amenorrea, hiperandrogenismo clínico o bioquímico y presencia de MOP, de acuerdo a lo que se detalla a continuación.

Hiperandrogenismo clínico: se utilizó el score de Ferriman para consignar la distribución y cuantificación del vello, se consideró un valor $\geq 8$.

Hiperandrogenismo bioquímico: valor de testosterona $>0,7 \mathrm{ng} / \mathrm{ml}$, androstenediona $>2,46$ $\mathrm{ng} / \mathrm{ml}$ o IAL $>6,0$; de acuerdo a valores de corte establecidos previamente en población chilena sana en nuestro laboratorio ${ }^{22}$.

Oligomenorrea: ciclos de más de 35 días.

Amenorrea: ausencia de menstruación por más de 3 meses, con test de embarazo negativo y nivel de progesterona $<4 \mathrm{ng} / \mathrm{ml}$.

MOP ecográfico: ovario con $\geq 12$ folículos (barrido completo) entre 2-9 $\mathrm{mm}$ de diámetro o un volumen $>10 \mathrm{ml}$. Sólo un ovario afectado fue suficiente para definir el síndrome. En presencia de folículo dominante (>10 mm) o cuerpo lúteo, el examen fue repetido en el siguiente ciclo.

Se excluyeron mujeres que presentaran otras causas de hiperandrogenismo, antecedente de diabetes mellitus y usuarias de medicamentos insulinosensibilizantes, anticonceptivos, y antiandrógenos 6 meses previos al estudio.

Todas las pacientes habían dado su consentimiento informado por haber participado en distintos protocolos de proyectos.

Se consideró como cambio de peso corporal, una disminución o aumento de al menos 5\% del peso corporal inicial, en un tiempo no mayor a 3 años desde la primera evaluación. Para la variable tiempo, se consideró un plazo de 5-8 años entre la evaluación inicial y final, sólo incluimos pacientes que no presentaron cambios significativos en el peso corporal. Finalmente, para evaluar el impacto del período embarazo-lactancia, se consideró un período entre 6 meses y 1 año desde el término de la lactancia.

\section{Ensayos utilizados}

Las determinaciones séricas de glucosa, insulina, testosterona, androstenediona, perfil lipídico y de la proteína transportadora de hormonas sexuales (SHBG) se realizaron de acuerdo a lo descrito previamente ${ }^{23}$.

Se calculó el índice de andrógenos libres (IAL), según la siguiente fórmula: Testosterona (nmol)/ SHBG (nmol) x $100^{24}$.

\section{Análisis estadístico}

Las variables continuas fueron descritas mediante percentiles, media y desviación estándar. Las diferencias entre los fenotipos se evaluaron por un test de ANOVA de un factor, seguido por la prueba de Bonferroni para comparaciones múltiples. Las diferencias en un mismo fenotipo entre el inicio y término del estudio fueron analizadas mediante la prueba de $t$ de Student o Mann-Whitney, según la distribución de los datos. La condición de cambio fenotípico desfavorable fue asociada con las posibles variables explicativas mediante un análisis de regresión logística binaria. Los intervalos de confianza son de nivel 95\% y se utilizó una significación de 5\%. Los datos fueron procesados en el programa STATA versión 12.0.

\section{Resultados}

La distribución porcentual inicial de los distintos fenotipos de SOP se muestra en la Figura 1-A. El 68,2\% de las mujeres presentó el fenotipo A; $19,3 \%$ el fenotipo B; $10,2 \%$ el fenotipo C y $2,3 \%$ el fenotipo D.

Como muestra la Figura 1-B, en la segunda evaluación se observa una distribución porcentual fenotípica distinta, cambio que fue significativo $\left(\chi^{2}=32,2, \mathrm{p}<0,001\right)$. Además, $18,2 \%$ de las pacientes ya no reunía los criterios diagnósticos de SOP.

En conjunto, 63,8\% de las pacientes modificó el fenotipo inicial de Rotterdam. El $64 \%$ de las mujeres que se embarazaron modificaron su fenotipo inicial, la disminución y alza del peso corporal provocaron un cambio fenotípico en 70\% y 56\% de las pacientes, respectivamente. Finalmente, el transcurso del tiempo generó un cambio fenotípico en $60 \%$ de las mujeres.

En este grupo de mujeres, 37,6\% experimentó una disminución de peso corporal, $23,9 \%$ un aumento del mismo, $14,7 \%$ el paso del tiempo y $23,9 \%$ un embarazo.

La Tabla 1 muestra las características clínicas de las mujeres con SOP de acuerdo a la clasificación fenotípica inicial y al término del estudio. En general, la edad, el IMC y los otros parámetros clínicos consignados fueron comparables entre los fenotipos al inicio del estudio. Al término del estudio, los fenotipos B y C, así como el grupo de mujeres que quedaron fuera de la clasificación de SOP mostraron un perfil antropométrico más favorable que el fenotipo A. 
$1-\mathrm{A}$

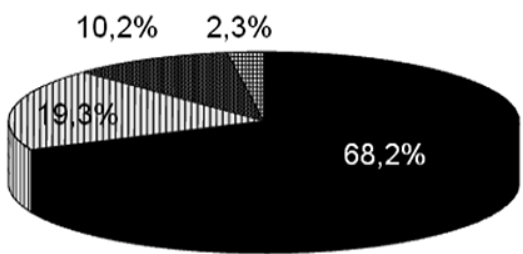

$1-B$

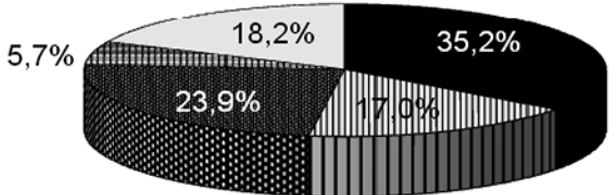

$\begin{array}{ll}\text {-Fenotipo A } & \text { ⿴囗十 } \text { Fenotipo D } \\ \text { mFenotipo B } & \square \text { No SOP } \\ \text { mFenotipo C } & \end{array}$

Figura 1. Clasificación inicial (1-A) y final (1-B) de los fenotipos de Rotterdam de síndrome de ovario poliquístico en mujeres chilenas en la primera evaluación y luego de distintas condiciones fisiológicas.

Tabla 1. Características clínicas de las pacientes con SOP, de acuerdo a la clasificación fenotípica de Rotterdam al inicio y término del estudio

\begin{tabular}{|c|c|c|c|c|c|}
\hline & $\mathbf{A}$ & B & C & D & No SOP \\
\hline Inicio del estudio & $(n=60)$ & $(n=17)$ & $(n=9)$ & $(n=2)$ & \\
\hline Edad (años) & $22,3 \pm 5,3$ & $24,9 \pm 7,3$ & $25,7 \pm 5,7$ & $24,5 \pm 14,8$ & - \\
\hline Peso $(\mathrm{kg})$ & $73,6 \pm 17,2$ & $71,2 \pm 12,4$ & $72,3 \pm 14,2$ & $62,5 \pm 3,5$ & - \\
\hline Talla (mt) & $1,58 \pm 0,05$ & $1,59 \pm 0,07$ & $1,55 \pm 0,06$ & $1,56 \pm 0,01$ & - \\
\hline $\mathrm{IMC}\left(\mathrm{kg} / \mathrm{m}^{2}\right)$ & $29,2 \pm 5,9$ & $27,8 \pm 3,9$ & $30,1 \pm 5,6$ & $25,5 \pm 1,2$ & - \\
\hline Cintura (cm) & $87,9 \pm 14,5$ & $89,2 \pm 13,4$ & $88,4 \pm 13,0$ & $72,0 \pm 0,01$ & - \\
\hline Cadera $(\mathrm{cm})$ & $103,0 \pm 14,9$ & $103,1 \pm 10,6$ & $99,3 \pm 12,6$ & $89,0 \pm 1,4$ & - \\
\hline ICC & $0,85 \pm 0,06$ & $0,86 \pm 0,05$ & $0,90 \pm 0,06$ & $0,81 \pm 0,01$ & - \\
\hline Score Ferriman & $12(7-17)$ & $15(11-19)$ & $13(7-18)$ & $5(4-6)$ & - \\
\hline Peso de nacimiento (g) & $3216 \pm 621$ & $3015 \pm 574$ & $2888 \pm 427$ & $3275 \pm 106$ & - \\
\hline Término del estudio & $(n=31)$ & $(n=15)$ & $(n=21)$ & $(n=5)$ & $(n=16)$ \\
\hline Edad (años) & $27,6 \pm 5,6^{*}$ & $28,0 \pm 6,7$ & $24,1 \pm 5,5^{b}$ & $24,6 \pm 8,1$ & $27,3 \pm 8,2$ \\
\hline Peso (kg) & $80,6 \pm 14,9$ & $66,6 \pm 12,2^{a}$ & $71,9 \pm 17,9$ & $80,5 \pm 29,2$ & $66,9 \pm 13,4^{d}$ \\
\hline Talla (mt) & $1,60 \pm 0,06$ & $1,58 \pm 0,05$ & $1,57 \pm 0,06^{b}$ & $1,56 \pm 0,03$ & $1,57 \pm 0,05^{d}$ \\
\hline $\mathrm{IMC}\left(\mathrm{kg} / \mathrm{m}^{2}\right)$ & $31,2 \pm 5,1$ & $26,8 \pm 4,4^{a}$ & $29,0 \pm 6,0$ & $33,7 \pm 13,0$ & $27,2 \pm 5,2^{d}$ \\
\hline Cintura $(\mathrm{cm})$ & $96,6 \pm 112,2^{*}$ & $82,0 \pm 10,4^{a}$ & $82,5 \pm 11,7^{b}$ & $91,3 \pm 18,8$ & $87,7 \pm 14,6$ \\
\hline Cadera $(\mathrm{cm})$ & $108,1 \pm 11,4$ & $95,1 \pm 11,1^{\mathrm{a}}$ & $98,3 \pm 9,2^{b}$ & $105,7 \pm 15,3$ & $101,9 \pm 11,2$ \\
\hline ICC & $0,89 \pm 0,08^{*}$ & $0,86 \pm 0,08$ & $0,84 \pm 0,08^{b}$ & $0,86 \pm 0,08$ & $0,86 \pm 0,06$ \\
\hline Score Ferriman & $16(4-29)$ & $16(6-23)$ & $12(7-17)$ & $5(4-6)$ & $9(5-14)^{d, e}$ \\
\hline Peso de nacimiento ( $\mathrm{g}$ ) & $3223 \pm 552$ & $2939 \pm 522$ & $3062 \pm 765$ & $3516 \pm 176$ & $3237 \pm 500$ \\
\hline
\end{tabular}

Valores expresados en promedio y desviación estándar. Score de Ferriman expresado en promedio y rango. Se consideró un $p<0,05$, como estadísticamente significativo. ${ }^{a} p<0,05$ al comparar entre fenotipo A y B. ${ }^{b} p<0,05$ al comparar entre fenotipo A y C. ${ }^{d} p<0,05$ al comparar entre fenotipo A y No SOP. ${ }^{e} p<0,05$ al comparar entre fenotipo B y No SOP. * $p<0,05$ al comparar cada fenotipo entre el inicio y término del estudio. 
La Tabla 2 presenta los parámetros metabólicos de los distintos fenotipos al inicio y término del estudio. Al inicio del estudio, la concentración de triglicéridos del fenotipo $\mathrm{C}$ fue estadísticamente menor, en comparación a los fenotipos $\mathrm{A}$ $(p=0,01)$ y $B(p=0,018)$. Al término del estudio, el fenotipo $B$ mostró mayor concentración de HDL que el fenotipo A ( $p=0,045)$; por su parte, el fenotipo $\mathrm{C}$ presentó una menor concentración de triglicéridos y de insulina en comparación al fenotipo $A(p=0,003$ y $p=0,006$, respectivamente). El grupo que perdió la condición SOP fue metabólicamente más saludable que el fenotipo A. Dado los pocos casos que presenta el fenotipo $\mathrm{D}$, este no se consideró en el análisis estadístico.
Al comparar los parámetros clínicos y metabólicos de cada fenotipo al inicio y término del estudio, sólo el fenotipo A presentó diferencias estadísticamente significativas entre ambas etapas, con mayor edad $(\mathrm{p}<0,0001)$, diámetro de cintura $(\mathrm{p}=0,002), \operatorname{ICC}(\mathrm{p}=0,01)$, concentración de LDL $(\mathrm{p}=0,047)$ y glucosa de postcarga $(\mathrm{p}=0,038)$ en el estado final. Los otros fenotipos no mostraron cambios significativos entre el inicio y el término del estudio.

La Tabla 3 muestra en detalle la movilidad de los fenotipos de acuerdo a cada condición estudiada. Se puede observar en cada grupo, cuantas pacientes permanecen en su clasificación fenotípica inicial y el sentido de la modificación.

Tabla 2. Características metabólicas de las pacientes con SOP, de acuerdo a la clasificación fenotípica de Rotterdam al inicio y término del estudio

\begin{tabular}{|c|c|c|c|c|c|}
\hline & $\mathbf{A}$ & B & C & D & No SOP \\
\hline Inicio del estudio & $(n=60)$ & $(n=17)$ & $(n=9)$ & $(n=2)$ & \\
\hline \multicolumn{6}{|l|}{ Ayuno } \\
\hline Glucosa (mg/dl) & $83,2 \pm 11,4$ & $88,7 \pm 9,5$ & $80,9 \pm 14,4$ & $69,0 \pm 14,4$ & - \\
\hline Insulina $(\mu \mathrm{Ul} / \mathrm{ml})$ & $18,2 \pm 12,5$ & $19,1 \pm 12,3$ & $15,1 \pm 10,3$ & $6,7 \pm 10,3$ & - \\
\hline Triglicéridos (mg/dl) & $157,8 \pm 60,9$ & $158,0 \pm 85,8$ & $93,2 \pm 19,2^{b, c}$ & $99,0 \pm 19,2$ & - \\
\hline Colesterol total (mg/dl) & $179,4 \pm 58,2$ & $204,8 \pm 46,7$ & $165,8 \pm 36,1$ & $116,0 \pm 36,1$ & - \\
\hline Colesterol HDL (mg/dl) & $37,7 \pm 13,8$ & $42,3 \pm 11,6$ & $39,4 \pm 11,5$ & $38,9 \pm 11,5$ & - \\
\hline Colesterol LDL (mg/dl) & $111,5 \pm 55,4$ & $130,6 \pm 36,6$ & $107,7 \pm 35,6$ & $57,3 \pm 35,6$ & - \\
\hline HOMA-IR & $3,8 \pm 2,5$ & $4,3 \pm 3,1$ & $3,1 \pm 2,4$ & $1,1 \pm 2,4$ & - \\
\hline \multicolumn{6}{|l|}{ Post-carga glucosa } \\
\hline Glucosa (mg/dl) & $97,1 \pm 23,9$ & $104,3 \pm 27,4$ & $106,1 \pm 34,2$ & $79,8 \pm 34,2$ & - \\
\hline Insulina ( $\mu \mathrm{UI} / \mathrm{ml})$ & $106,2 \pm 70,7$ & $112,8 \pm 88,6$ & $82,1 \pm 131,2$ & $67,6 \pm 131,2$ & - \\
\hline ISIcomposite & $4,2 \pm 2,6$ & $3,6 \pm 1,9$ & $5,4 \pm 2,7$ & $8,1 \pm 1,3$ & - \\
\hline Término del estudio & $(n=31)$ & $(n=15)$ & $(n=21)$ & $(n=5)$ & $(n=16)$ \\
\hline \multicolumn{6}{|l|}{ Ayuno } \\
\hline Glucosa (mg/dl) & $88,9 \pm 12,4$ & $81,2 \pm 12,3$ & $82,0 \pm 6,7$ & $83,2 \pm 9,2$ & $89,8 \pm 9,6^{\dagger}$ \\
\hline Insulina ( $\mu \mathrm{UI} / \mathrm{ml})$ & $26,0 \pm 16,6$ & $16,0 \pm 13,1$ & $18,7 \pm 22,5$ & $9,8 \pm 4,4$ & $10,7 \pm 4,1^{d}$ \\
\hline Triglicéridos (mg/dl) & $192,7 \pm 88,9$ & $146,4 \pm 110,4$ & $95,0 \pm 35,4^{b}$ & $82,0 \pm 21,4$ & $130,7 \pm 40,2$ \\
\hline Colesterol total (mg/dl) & $224,0 \pm 78,2$ & $180,5 \pm 66,9$ & $179,3 \pm 34,3$ & $151,0 \pm 30,4$ & $166,7 \pm 25,6$ \\
\hline Colesterol HDL (mg/dl) & $34,7 \pm 8,2$ & $44,6 \pm 12,5^{a}$ & $41,9 \pm 9,3$ & $41,0 \pm 9,7,5$ & $46,4 \pm 13,6^{d}$ \\
\hline Colesterol LDL (mg/dl) & $153,3 \pm 68,2^{*}$ & $111,5 \pm 70,8$ & $116,7 \pm 31,8$ & $93,6 \pm 25,3$ & $94,1 \pm 16,6$ \\
\hline HOMA-IR & $5,7 \pm 4,7$ & $3,5 \pm 3,4$ & $2,6 \pm 1,5^{b}$ & $2,0 \pm 0,8$ & $2,4 \pm 0,9^{d}$ \\
\hline \multicolumn{6}{|l|}{ Post-carga glucosa } \\
\hline Glucosa (mg/dl) & $110,0 \pm 26,3^{*}$ & $116,0 \pm 35,2$ & $98,9 \pm 17,1$ & $120,2 \pm 14,6$ & $90,8 \pm 16,9^{d}$ \\
\hline Insulina ( $\mu \mathrm{UI} / \mathrm{ml})$ & $137,8 \pm 115,6$ & $92,8 \pm 75,1$ & $62,1 \pm 40,3^{b}$ & $127,3 \pm 115,1$ & $41,9 \pm 29,5^{\mathrm{d}, \mathrm{e}}$ \\
\hline ISIcomposite & $3,7 \pm 2,9$ & $5,7 \pm 3,8$ & $6,1 \pm 3,6^{b}$ & $4,2 \pm 1,6$ & $6,2 \pm 3,4^{d}$ \\
\hline
\end{tabular}

Valores expresados en promedio y desviación estándard. Se consideró un $p<0,05$, como estadísticamente significativo. ${ }^{a} p<0,05$ al comparar entre fenotipo A y B. ${ }^{b} p<0,05$ al comparar entre fenotipo A y C. ${ }^{c} p<0,05$ al comparar entre fenotipo B y C. ${ }^{d} p<0,05$ al comparar entre fenotipo A y No SOP. e $p<0,05$ al comparar entre fenotipo B y No SOP. ${ }^{f} p<0,05$ al comparar entre fenotipo $C$ y No SOP. ${ }^{*} p<0,05$ al comparar cada fenotipo entre el inicio y término del estudio. 
Tabla 3. Movilidad fenotípica en las pacientes con SOP de acuerdo a la condición estudiada, al inicio y término del estudio

\begin{tabular}{|c|c|c|c|c|c|c|c|}
\hline & \multirow{2}{*}{\multicolumn{2}{|c|}{ Fenotipo inicial (n) }} & \multicolumn{5}{|c|}{ Fenotipo final (n) } \\
\hline & & & A & B & C & D & No SOP \\
\hline \multicolumn{8}{|c|}{ Embarazo } \\
\hline & A & 16 & 7 & 1 & 4 & 1 & 3 \\
\hline & B & 3 & 2 & 1 & - & - & - \\
\hline & C & 2 & 1 & - & - & - & 1 \\
\hline & D & - & - & - & - & - & - \\
\hline & No SOP & - & - & - & - & - & - \\
\hline Total & & 21 & 10 & 2 & 4 & 1 & 4 \\
\hline \multicolumn{8}{|c|}{ Baja peso } \\
\hline & A & 21 & 3 & 3 & 10 & 1 & 4 \\
\hline & B & 8 & - & 3 & 1 & - & 4 \\
\hline & C & 3 & - & 1 & 1 & - & 1 \\
\hline & D & 1 & - & - & - & 1 & - \\
\hline & No SOP & - & - & - & - & - & - \\
\hline Total & & 33 & 3 & 7 & 12 & 2 & 9 \\
\hline \multicolumn{8}{|c|}{ Alza peso } \\
\hline & A & 15 & 10 & 2 & 1 & 1 & 1 \\
\hline & B & 3 & 2 & - & 1 & - & - \\
\hline & C & 2 & 1 & - & 1 & - & - \\
\hline & D & 1 & - & - & - & 1 & - \\
\hline & No SOP & - & - & - & - & - & - \\
\hline Total & & 21 & 13 & 2 & 3 & 2 & 1 \\
\hline \multicolumn{8}{|c|}{ Paso tiempo } \\
\hline & A & 8 & 3 & 3 & 1 & - & 1 \\
\hline & B & 3 & 1 & 1 & 1 & - & - \\
\hline & C & 2 & 1 & - & - & - & 1 \\
\hline & D & - & - & - & - & - & - \\
\hline & No SOP & - & - & - & - & - & - \\
\hline Total & & 13 & 5 & 4 & 2 & - & 2 \\
\hline
\end{tabular}

$\mathrm{Al}$ analizar el impacto de cada una de las condiciones estudiadas, sólo la disminución del peso corporal tendría un efecto estadísticamente significativo $(\mathrm{p}=0,047)$ indicando movilidad hacia un fenotipo más favorable. En promedio, este grupo disminuyó en $11,4 \%$ su peso corporal inicial, lo que correspondió a 2,5 puntos de IMC $(6,4 \mathrm{~kg})$.

$\mathrm{Al}$ tomar todas las condiciones en conjunto, el impacto más relevante en la movilidad fenotípica fue dado por el cambio de peso. Hay evidencia de que una disminución del peso corporal propicia un cambio favorable en la movilidad fenotípica $(\mathrm{p}=0,05)$. Visto en el sentido opuesto, un aumento de peso en 1 punto de IMC, confiere un riesgo de $8 \%$ más de pasar hacia un fenotipo más desfavorable, independientemente del peso corporal inicial.
El transcurso del tiempo y el embarazo-lactancia no mostraron un efecto concluyente en cuanto a la movilidad fenotípica.

\section{Discusión}

Desde la descripción de los fenotipos de Rotterdam, se ha planteado la disyuntiva de si existen diferencias en la severidad de las manifestaciones de SOP entre los distintos fenotipos y si esta clasificación se mantiene a lo largo de la vida.

En el presente estudio quisimos evaluar si condiciones fisiológicas podrían afectar los fenotipos de Rotterdam del SOP. Nuestros resultados muestran que la clasificación inicial de un fenotipo no es estática y se modifica por las condiciones 
Efecto de condiciones fisiológicas sobre los fenotipos de SOP - B. Echiburú et al

estudiadas. Si este cambio tiene un impacto favorable o desfavorable, depende de cada condición en particular.

Semejante a lo descrito en estudios previos, nuestros resultados muestran que el fenotipo más prevalente es el $\mathrm{A}^{25}$, siendo el $\mathrm{D}$ el menos frecuente en esta población estudiada.

Respecto a los rasgos metabólicos, nuestros resultados sugieren que los fenotipos A y B serían los más afectados, lo que es similar a lo descrito por otros autores ${ }^{25}$. Estos fenotipos presentaron una mayor concentración de triglicéridos, lo cual fue estadísticamente significativo. Además, la sensibilidad insulínica evaluada mediante el HOMA-IR y el ISI composite, si bien fue diferentes entre los grupos A y B respecto al C, no alcanzó significancia estadística por la dispersión de los datos.

En la mujer normal, el paso del tiempo confiere algunos cambios fisiológicos de su esfera reproductiva, como disminución en la producción de andrógenos, del volumen ovárico y número de folículos.

En este sentido, algunos estudios han sugerido que las manifestaciones clínicas y bioquímicas del SOP podrían atenuarse con la edad ${ }^{26}$. Los ciclos menstruales tienden a regularizarse ${ }^{27}$ y el hiperandrogenismo se reduce ${ }^{28}$. Tal como en mujeres sanas, disminuye el número de folículos y el volumen ovárico ${ }^{29}$. En mujeres con SOP los cambios relacionados con la edad dificultan la identificación de pacientes no diagnosticadas en la edad reproductiva temprana, lo que podría afectar la incidencia observada de $\mathrm{SOP}^{30}$.

En el presente estudio, luego de 5-8 años de la primera evaluación, si bien hubo movilidad fenotípica, esta no fue homogénea; probablemente porque las pacientes se mantuvieron en un rango etario comparable. Nuestros datos difieren de lo descrito por otros autores, que observaron una disminución en la severidad de las manifestaciones del SOP en seguimientos a largo plazo. Carmina et al, en un seguimiento por 20 años en mujeres con SOP diagnosticadas en la edad reproductiva temprana, observaron que luego de 10 años comenzaba progresivamente a disminuir la concentración de andrógenos y el volumen ovárico y los ciclos menstruales se hacían más regulares y ovulatorios ${ }^{18}$. Lo que concuerda con lo observado por otros autores ${ }^{16,30}$.

Aunque la obesidad no está considerada en la definición del $\mathrm{SOP}^{31}$, la importancia de ella se evidencia por el impacto que tiene una discreta pérdida de peso $(5 \%)$ en disminuir algunas manifestaciones de hirsutismo ${ }^{32-34}$, regularizar los ciclos menstruales y la ovulación, así como mejorar otras anormalidades endocrinas y metabólicas ${ }^{32,35,36}$. Más aún, una baja de peso mayor ( 5 puntos de IMC, equivalente a14 $\mathrm{kg}$ ) se traduce en que el $36,9 \%$ de las pacientes ya no reúnen los criterio de $\mathrm{SOP}^{17}$.

En nuestro estudio observamos que la disminución del peso corporal tendría un efecto beneficioso en la expresión del SOP, promoviendo un cambio hacia un fenotipo menos severo. El hecho de que el alza en un punto de IMC confiera mayor riesgo de pasar a un fenotipo más afectado, es un hecho importante a considerar en el momento de informar a estas pacientes.

Un aspecto novedoso evaluado en este estudio, fue el impacto que el período embarazo-lactancia podría tener en la movilidad fenotípica.

Es sabido que el embarazo per se constituye un estado fisiológico de insulino resistencia, lo que a su vez podría contribuir a un estado hiperandrogénico. En estudios previos establecimos que las mujeres embarazadas con SOP presentan elevadas concentraciones de andrógenos, insulina y triglicéridos, menores concentraciones de adiponectina y una exacerbación de la resistencia insulínica fisiológica del embarazo ${ }^{37,38}$. A su vez, la lactancia en estas mujeres, reduciría transitoriamente la concentración de insulina y otros marcadores biológicos de resistencia insulínica ${ }^{39,40}$.

Por lo tanto, como producto de lo anterior, es posible que durante el período que sigue a la lactancia algunas funciones metabólicas y endocrinas del SOP pudieran ser transitoriamente modificadas.

De hecho, en este estudio pudimos observar que más de la mitad de las mujeres modificó su fenotipo inicial en el período posterior al amamantamiento. Sin embargo, por el momento no podemos decir si la resultante de este período es favorable o desfavorable, por lo que se requiere de un mayor número de casos.

De acuerdo a nuestras observaciones, la clasificación de los fenotipos de Rotterdam no es estática, ya que diversas condiciones fisiológicas que ocurren durante la vida reproductiva de la mujer podrían provocar un cambio fenotípico. Nuestros resultados son categóricos en establecer que la disminución del peso corporal podría ser 
favorable y llevaría hacia fenotipos menos severos. El efecto del paso del tiempo, no tuvo un resultado concluyente, debido a que las pacientes no cambiaron de etapa reproductiva. Uno de los aspectos más novedosos de nuestra investigación fue el estudio de las consecuencias del embarazolactancia, sin embargo, por el momento no obtuvimos resultados concluyentes.

En síntesis, el SOP es una condición en la cual se han descrito factores genéticos y epigenéticos, cuya expresión puede ser catalogada en fenotipos con distinto grado de penetrancia. Las condiciones fisiológicas estudiadas generan una movilidad fenotípica, que incluso puede modificar la expresión de este síndrome a tal grado que en un porcentaje no despreciable de pacientes, éste ya no sea reconocible.

Agradecimientos: Deseamos agradecer a los miembros académicos y no académicos del Laboratorio de Endocrinología y Metabolismo de la Facultad de Medicina Occidente de la Universidad de Chile por su colaboración. Queremos agradecer además, al Dr. Gabriel Cavada, Ph.D., del Departamento de Salud Pública, Facultad de Medicina, Universidad de Chile, por su ayuda en el análisis estadístico de este estudio.

\section{Referencias}

1. Livadas S, Diamanti-Kandarakis E. Polycystic ovary syndrome: definitions, phenotypes and diagnostic approach. Front Horm Res 2013; 40: 1-21.

2. Azziz R, Carmina E, Dewailly D, Diamanti-Kandarakis E, Escobar-Morreale HF, Futterweit W, et al. The Androgen Excess and PCOS Society criteria for the polycystic ovary syndrome: the complete task force report. Fertil Steril 2009; 91 (2): 456-88.

3. Lunde O, Magnus P, Sandvik L, Høglo S. Familial clustering in the polycystic ovarian syndrome. Gynecol Obstet Invest 1989; 28 (1): 23-30.

4. Luque-Ramírez M, San Millán JL, Escobar-Morreale HF. Genomic variants in polycystic ovary syndrome. Clin Chim Acta 2006; 366 (1-2): 14-26.

5. Abbott DH, Dumesic DA, Franks S. Developmental origin of polycystic ovary syndrome-a hypothesis. J Endocrinol 2002; 174 (1): 1-5.

6. Diamanti-Kandarakis E, Christakou C, Palioura E, Kandaraki E, Livadas S. Does polycystic ovary syndrome start in childhood? Pediatr Endocrinol Rev 2008; 5 (4): 904-11.
7. Stein I, Leventhal M. Amenorrhea associated with bilateral polycystic ovaries. Am. J. Obstet Gynecol 1935; 29: 181-91.

8. Zawadski JK, Dunaif A. Diagnostic criteria for polycystic ovary syndrome: towards a rationale approach. En: Polycystic Ovary Syndrome, ed. por Dunaif A, Givens J R, Haseltine F, Merriam G R, Boston: Blackwell 1992: 377-84.

9. Rotterdam ESHRE/ASRM-Sponsored PCOS Consensus Workshop Group. Revised 2003 consensus on diagnostic criteria and long-term health risks related to polycystic ovary syndrome. Fertil Steril 2004; 81 (1): 19-25.

10. Azziz R, Carmina E, Dewailly D, Diamanti-Kandarakis E, Escobar-Morreale HF, Futterweit W, et al. Positions statement: criteria for defining polycystic ovary syndrome as a predominantly hyperandrogenic syndrome: an Androgen Excess Society guideline. J Clin Endocrinol Metab 2006; 91 (11): 4237-45.

11. Carmina E, Chu MC, Longo RA, Rini GB, Lobo RA. Phenotypic variation in hyperandrogenic women influences the findings of abnormal metabolic and cardiovascular risk parameters. J Clin Endocrinol Metab 2005; 90 (5): 2545-9.

12. Yilmaz M, Isaoglu U, Delibas IB, Kadanali S. Anthropometric, clinical and laboratory comparison of four phenotypes of polycystic ovary syndrome based on Rotterdam criteria. J Obstet Gynaecol Res 2011; 37 (8): 1020-6.

13. Wang Y, Qu J, Wu X, Hou L, Erkkola R, Wang Y. Different phenotypes of polycystic ovary syndrome by Rotterdam criteria are differently steroidogenic but similarly insulin resistant. Fertil Steril 2010; 93 (4): 1362-5.

14. Panidis D, Tziomalos K, Misichronis G, Papadakis E, Betsas G, Katsikis I, et al. Insulin resistance and endocrine characteristics of the different phenotypes of polycystic ovary syndrome: a prospective study. Hum Reprod 2012; 27 (2): 541-9.

15. Liou TH, Yang JH, Hsieh CH, Lee CY, Hsu CS, Hsu MI. Clinical and biochemical presentations of polycystic ovary syndrome among obese and nonobese women. Fertil Steril 2009; 92 (6): 1960-5.

16. Panidis D, Tziomalos K, Macut D, Delkos D, Betsas G, Misichronis G, et al. Cross-sectional analysis of the effects of age on the hormonal, metabolic, and ultrasonographic features and the prevalence of the different phenotypes of polycystic ovary syndrome. Fertil Steril 2012; 97 (2): 494-500.

17. Pasquali R, Gambineri A, Cavazza C, Ibarra Gasparini D, Ciampaglia W, Cognigni GE, et al. Heterogeneity in the responsiveness to long-term lifestyle intervention and predictability in obese women with polycystic ovary syndrome. Eur J Endocrinol 2011; 164 (1): 53-60. 
18. Carmina E, Campagna AM, Lobo RA. A 20-year followup of young women with polycystic ovary syndrome. Obstet Gynecol 2012; 119 (2 Pt 1): 263-9.

19. Ferriman D, Gallwey JD. Clinical assessment of body hair growth in women. J Clin Endocrinol Metab1961; 21: 1440-7.

20. Matthews DR, Hosker JP, Rudenski AS, Naylor BA, Treacher DF, Turner RC. Homeostasis model assessment: insulin resistance and beta-cell function from fasting plasma glucose and insulin concentrations in man. Diabetologia 1985; 28 (7): 412-9.

21. Matsuda M, De Fronzo RA. Insulin sensitivity indices obtained from oral glucose tolerance testing: comparison with the euglycemic insulin clamp. Diabetes Care 1999; 22 (9): 1462-70.

22. Maliqueo M, Atwater I, Lahsen R, Pérez-Bravo F, Angel B, Sir-Petermann T. Proinsulin serum concentrations in women with polycystic ovary syndrome: a marker of beta-cell dysfunction? Hum Reprod 2003; 18 (12): 2683 8.

23. Echiburú B, Pérez-Bravo F, Maliqueo $M$, Sánchez $F$, Crisosto N, Sir-Petermann T. Polymorphism T --> C (-34 base pairs) of gene CYP17 promoter in women with polycystic ovary syndrome is associated with increased body weight and insulin resistance: a preliminary study. Metabolism 2008; 57 (12): 1765-71.

24. Fox R, Corrigan E, Thomas PA, Hull MG. The diagnosis of polycystic ovaries in women with oligo-amenorrhoea: predictive power of endocrine tests. Clin Endocrinol (Oxf) 1991; 34 (2): 127-31.

25. Moghetti P, Tosi F, Bonin C, Di Sarra D, Fiers T, Kaufman JM, et al. Divergences in Insulin Resistance Between the Different Phenotypes of the Polycystic Ovary Syndrome. J Clin Endocrinol Metab 2013; 98 (4): 628-37.

26. Pasquali R, Gambineri A. Polycystic ovary syndrome: a multifaceted disease from adolescence to adult age. Ann N Y Acad Sci 2006; 1092: 158-74.

27. Elting MW, Korsen TJ, Rekers-Mombarg LT, Schoemaker J. Women with polycystic ovary syndrome gain regular menstrual cycles when ageing. Hum Reprod 2000; 15 (1): 24-8.

28. Winters SJ, Talbott E, Guzick DS, Zborowski J, McHugh KP. Serum testosterone levels decrease in middle age in women with the polycystic ovary syndrome. Fertil Steril 2000; 73 (4): 724-9.

29. Alsamarai S, Adams JM, Murphy MK, Post MD, Hayden DL, Hall JE, et al. Criteria for polycystic ovarian mor- phology in polycystic ovary syndrome as a function of age. J Clin Endocrinol Metab 2009; 94 (12): 4961-70.

30. Hsu MI. Changes in the PCOS phenotype with age. Steroids 2013; 78 (8): 761-6.

31. Ehrmann DA. Polycystic ovary syndrome. N Engl J Med 2005; 352 (12): 1223-36. Review.

32. Kiddy DS, Hamilton-Fairley D, Bush A, Short F, Anyaoku V, Reed MJ, et al. Improvement in endocrine and ovarian function during dietary treatment of obese women with polycystic ovary syndrome. Clin Endocrinol 1992; 36 (1): 105-11.

33. Pasquali R, Gambineri A, Pagotto U. The impact of obesity on reproduction in women with polycystic ovary syndrome. BJOG 2006; 113 (10): 1148-59.

34. Morán LJ, Pasquali R, Teede HJ, Hoeger KM, Norman RJ. Treatment of obesity in polycystic ovary syndrome: a position statement of the Androgen Excess and Polycystic Ovary Syndrome Society. Fertil Steril 2009; 92 (6): 1966-82.

35. Guzick DS, Wing R, Smith D, Berga SL, Winters SJ. Endocrine consequences of weight loss in obese, hyperandrogenic, anovulatory women. Fertil Steril 1994; 61 (4): 598-604.

36. Holte J, Bergh T, Berne C, Wide L, Lithell H. Restored insulin sensitivity but persistently increased early insulin secretion after weight loss in obese women with polycystic ovary syndrome. J Clin Endocrinol Metab 1995; 80 (9): 2586-93.

37. Sir-Petermann T, Maliqueo M, Angel B, Lara HE, PérezBravo F, Recabarren SE. Maternal serum androgens in pregnant women with polycystic ovarian syndrome: possible implications in prenatal androgenization. Hum Reprod 2002; 17 (10): 2573-9.

38. Sir-Petermann T, Echiburú B, Maliqueo M, Crisosto N, Sánchez F, Hitschfeld C, et al. Serum adiponectin and lipid concentrations in pregnant women with polycystic ovary syndrome. Hum Reprod 2007; 22 (7): 1830-6.

39. Maliqueo M, Sir-Petermann T, Salazar G, Pérez-Bravo F, Recabarren SE, Wildt L. Resumption of ovarian function during lactational amenorrhoea in breastfeeding women with polycystic ovarian syndrome: metabolic aspects. Hum Reprod 2001; 16 (8): 1598-602.

40. Sir-Petermann T, Devoto L, Maliqueo M, Peirano P, Recabarren SE, Wildt L. Resumption of ovarian function during lactational amenorrhoea in breastfeeding women with polycystic ovarian syndrome: endocrine aspects. Hum Reprod 2001; 16 (8): 1603-10. 